\title{
MICROSTRUCTURAL UNDERPINNINGS AND MACROSCALE FUNCTIONAL IMPLICATIONS OF TEMPORAL LOBE CONNECTIVITY GRADIENTS
}

Reinder Vos de Wael $^{1}$, Jessica Royer ${ }^{1}$, Shahin Tavakol ${ }^{1}$, Yezhou Wang ${ }^{1}$, Casey Paquola ${ }^{1}$, Oualid Benkarim $^{1}$, Nicole Eichert ${ }^{2}$, Sara Larivière ${ }^{1}$, Bratislav Misic ${ }^{1}$, Jonathan Smallwood ${ }^{3}$, Sofie L. Valk $^{4}$, Boris C. Bernhardt ${ }^{1}$

${ }^{1}$ Montreal Neurological Institute, McGill University, Montreal, Quebec, Canada $;{ }^{2}$ University of Oxford, Oxford, United Kingdo; ${ }^{3}$ Queens University, Kingston, Ontario, Canada; ${ }^{4}$ Max Planck Institute for Human Cognitive and Brain Sciences, Leipzig, Germany;

\section{CORRESPONDENCE TO}

Boris C. Bernhardt, PhD

McConnell Brain Imaging Centre,

Montreal Neurological Institute (NW-256), McGill University,

3801 Rue University, Montréal, QC, H3A 2B4, Canada

p. $(+1) 5143983579$

e. boris.bernhardt@mcgill.ca

w. mica-mni.github.io

Reinder Vos de Wael, MSc

e. reinder.vosdewael@mail.mcgill.ca 


\begin{abstract}
The temporal lobe is implicated in higher cognitive processes and is one of the regions that underwent substantial reorganization during primate evolution. Its functions are instantiated, in part, by its complex layout of structural connections. This study identified low-dimensional representations of structural connectivity variations in human temporal cortex and explored their microstructural underpinnings and associations to macroscale function. We identified three eigenmodes which described gradients in structural connectivity. These gradients reflected interregional variations in cortical microstructure derived from quantitative MRI and post-mortem histology. Gradient-informed models accurately predicted macroscale measures of temporal lobe function. Gradients aligned closely with established measures of functional reconfiguration and areal expansion between macaques and humans, highlighting the important role evolution has played in shaping temporal lobe function. Our results provide robust evidence for three axes of structural connectivity in human temporal cortex with consistent microstructural underpinnings and contributions to large-scale brain network function.
\end{abstract}




\section{INTRODUCTION}

The human temporal lobe is involved in multiple cognitive, affective, and sensory processes, including memory (Vaz et al., 2019), emotional reactivity (Phelps, 2004), semantic cognition (Ralph et al., 2017), as well as auditory processing (Bonilha et al., 2017). Notably, temporal lobe subregions have been suggested to serve as origins of major organizational and evolutionary axes of the human brain (Goulas et al., 2019; Sanides, 1969), and host structures, such as the middle and superior temporal gyri, that have undergone accelerated functional reconfigurations and areal expansion in primate evolution (Eichert et al., 2020; Mars, Sotiropoulos, et al., 2018; Xu et al., 2020). Collectively, these different aspects suggest that the temporal lobe is a hub implicated in important features of human cognition, and that its study may provide key insights into cortical organization and its phylogenetic basis.

In an attempt to understand the role of the temporal lobe in whole-brain networks, prior studies in non-human animals and human participants have started to delineate the complex connectivity profiles of the temporal lobe. Tract tracing studies in non-human primates have charted short range connections as well as long range tracts of the temporal lobe (Webster et al., 1991), showing distributed connectivity patterns to a diverse territory of cytoarchitectural areas (Beul et al., 2017; Mohedano-Moriano et al., 2015; Morán et al., 1987; Sakata et al., 2019). These findings were complemented by diffusion MRI tractography studies in both non-human primates (Bryant et al., 2020) and humans (Saur et al., 2008), where this non-invasive technique can approximate the course of white matter fiber tracts both in vivo and ex vivo. Diffusion MRI studies have been performed for all major long range fiber bundles (Howells et al., 2018; Roumazeilles et al., 2020; Smiley \& Falchier, 2009), for short range fiber systems (Attar et al., 2020) as well as the superficial white matter (Bodin et al., 2020; Hong, Hyung, et al., 2019; Liu et al., 2016; Oishi et al., 2008).

Beyond the mapping of specific fiber bundles, recent years have seen a shift towards the application of unsupervised approaches that identify and visualize low dimensional eigenmodes in connectivity changes across the cortical mantle - also referred to as connectivity gradients (Huntenburg et al., 2018; Margulies et al., 2016). A gradient perspective describes transitions of brain connectivity in a continuous reference frame, which has been proposed to capture subregional heterogeneity as well as functional multiplicity better than techniques that parcellate cortex into discrete subregions and average potentially variable properties within parcels (Bijsterbosch et al., 2020; Haak \& Beckmann, 2020; Mars, Sotiropoulos, et al., 2018). Capitalizing 
on resting-state functional MRI acquisitions, gradient mapping techniques have previously identified principal axes of neural organization in healthy adults and in non-human primates (Buckner \& Margulies, 2019; Guell et al., 2018; Haak et al., 2018; Margulies et al., 2016; Vos de Wael et al., 2018; Xu et al., 2020), and these techniques are increasingly used to study lifespan processes related to aging (Lowe et al., 2019; Bethlehem et al., 2020) and typical as well as atypical childhood development (Ball, Seidlitz, Beare, et al., 2020; Ball, Seidlitz, O’Muircheartaigh, et al., 2020; Hong, Vos de Wael, et al., 2019; Larivière et al., 2019; Park, Hong, et al., 2020). In the temporal lobe, these techniques have previously been applied to structural connectivity information, with the goal of subsequent parcellation (Bajada et al., 2017), to describe the ventral and anterior temporal lobe as a structural connectivity convergence zone (Bajada et al., 2019), and to relate structural connectivity gradients to meta-analytic task activations (Blazquez Freches et al., 2020; Yarkoni et al., 2011).

In the current work, we expanded on these previous findings in three ways:

(i) We explored regional associations between structural connectivity gradients in the temporal lobe and measures of intracortical microstructure to assess whether large scale connectivity axes are reflected in the local microcircuits. Prior findings in non-human animals suggest that an area's cytoarchitectonic properties may be predictive of structural connectivity, but precise associations between both remain underspecified in humans (Barbas, 2015). To fill this gap, our project leveraged both myelin sensitive MRI contrasts as well as post-mortem cytoarchitecture analysis based on BigBrain (Amunts et al., 2013).

(ii) Structural connectivity is generally assumed to constrain functional connectivity (Deco et al., 2017; Honey et al., 2009; Suárez et al., 2020; Wang et al., 2019). Here we assessed whether structural connectome gradients within the temporal lobe, as a low dimensional representation of structural connectivity, can predict intrinsic functional organization derived from resting-state fMRI acquisitions, both with respect to macroscale functional motifs as well as node-wise estimates of functional connectivity.

(iii) Finally, to determine phylogenetic principles of structural connectome organization, we examined whether structural connectivity gradients reflect principal dimensions of primate evolution. To this end, we studied the relationship of gradients with areal expansion and functional reconfigurations from non-human primates to humans (Xu et al., 2020). 
Our approach capitalized on multimodal image processing and advanced diffusion tractography analyses. Specifically, we leveraged a high-resolution representation of temporal lobe structural connectivity to resolve subregional heterogeneity in connectivity and multiplicity of potentially overlapping gradients. Our findings were replicated both in a hold-out dataset from the same site, and in a dataset acquired with a different scanner, imaging parameters, and preprocessing pipeline. We have released all codes to replicate the main figures on https:/github.com/MICA$\mathrm{MNI} /$ micaopen

\section{RESUltS}

Our main analyses were based on 75 unrelated participants of the Human Connectome Project (HCP) S900 release (Van Essen et al., 2013), a large-scale open-access neuroimaging dataset comprised of healthy young adults (HCP-Discovery; $\mathrm{n}=75$; age=29.2 \pm 3.6 , female=47). We also replicated all findings in a subset of unrelated participants from HCP, (HCP-Replication; $\mathrm{n}=75$; age $=28.9 \pm 4.0$, female $=44$ ). For each participant, we mapped structural connectivity of each vertex in the gray-white matter interface of the temporal lobe to the entire cortex using high resolution tractography (see Methods, for details). To identify structural connectivity gradients, we used nonlinear dimensionality reduction techniques that identify spatial eigenvectors explaining interregional variations in structural connectivity (Coifman \& Lafon, 2006). To assess the reproducibility of our findings, we repeated our analyses on the Microstructure Informed Connectomics (MICs) dataset, a separate dataset of healthy controls who underwent 3T imaging comparable to the HCP protocol in our center (54 controls, 30.5+7.3 years old, 20 females).

\section{Multiple gradients of structural connectivity in the temporal lobe}

In $H C P$-Discovery, the first three components of temporal cortical gradients collectively explained 67\% of variance in temporal lobe structural (Fig. 1Error! Reference source not found.A). Findings were similar in the left and right hemispheres. We thus present only left hemisphere results in the 

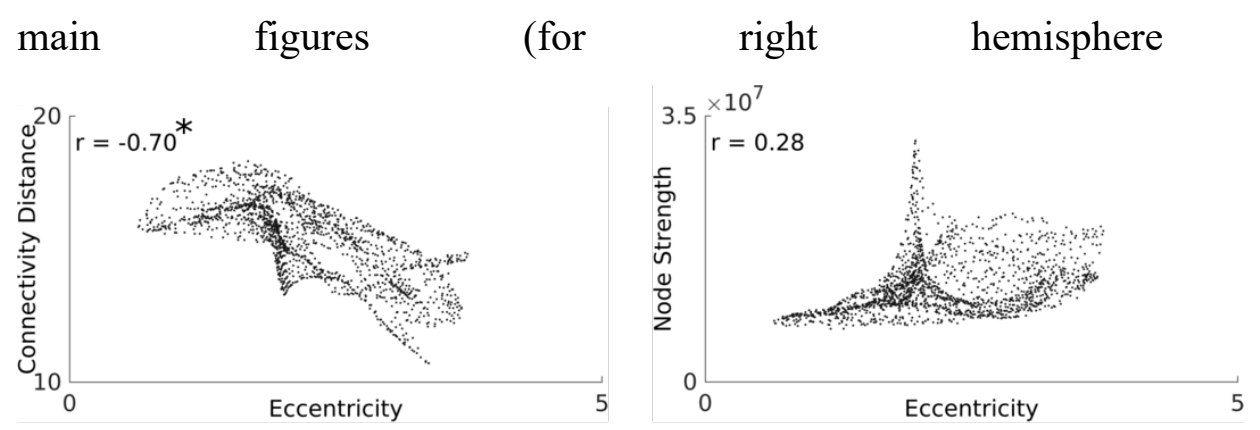

results,

see
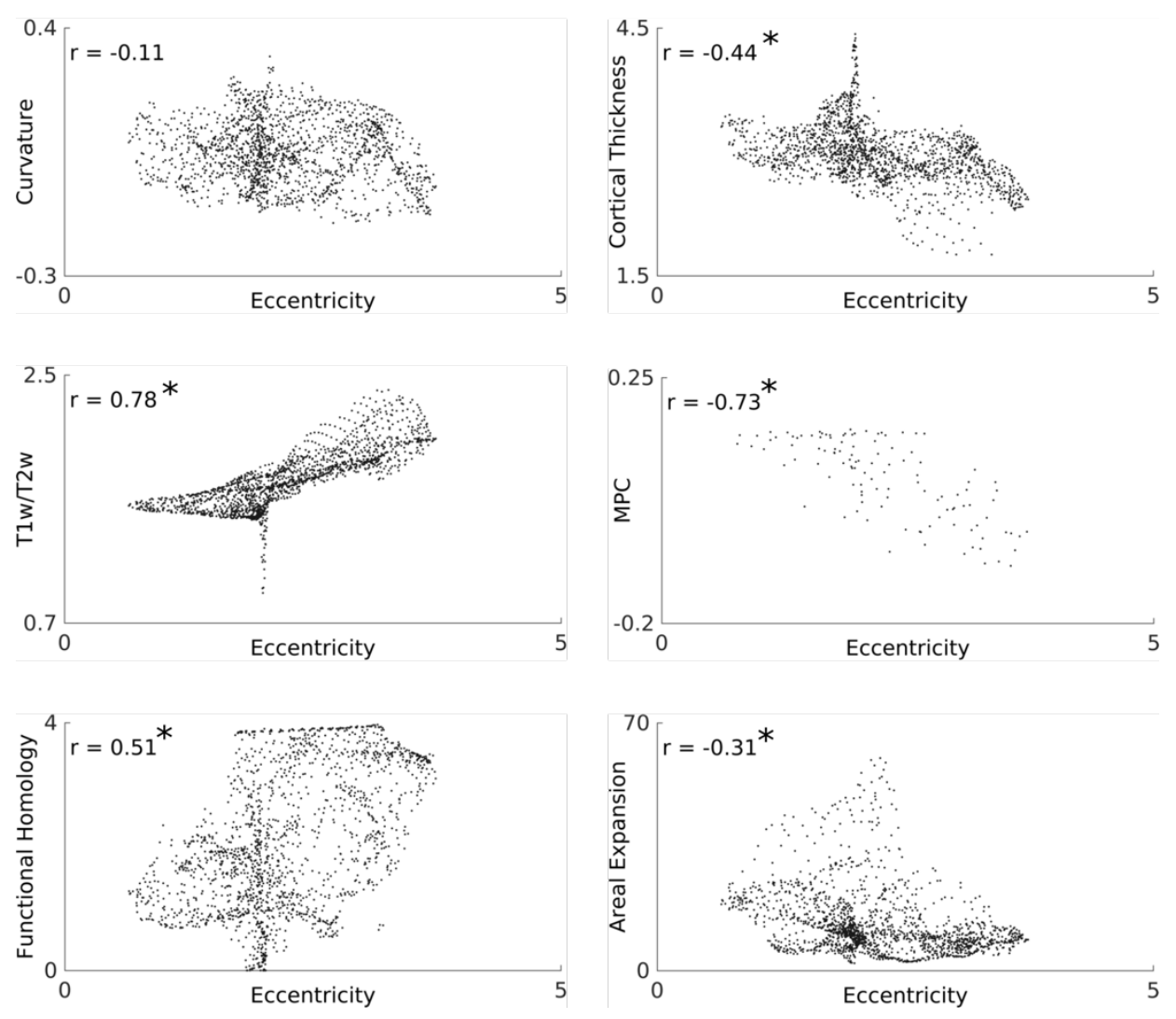

Supplementary Fig. 1). Gradient solutions were consistent across the different datasets studied, with absolute correlations between G1-3 of HCP-Discovery with G1-3 of HCP-Replication and MICs exceeding $\mathrm{r}>0.96$.

The first structural connectivity gradient (G1) ran between the superior temporal gyrus and the medial temporal lobe (Fig. 1Error! Reference source not found.A), the second (G2) along the posterior-anterior axis, and the third (G3) from anterolateral to posteromedial. To determine the connectivity patterns represented by each gradient, we mapped the connectivity of the top/bottom $10 \%$ of vertices of each gradient and assessed changes in the spatial distribution of connectivity profiles at the anchors of each gradient. G1 connectivity changes differentiated between visual and 
parietal connectivity, G2 involved changes from temporal pole and insula to visual/parietal cortex and lateral frontal cortex, and G3 described changes from visual/parietal to lateral temporal and frontal (Supplementary Fig. 2).

\section{A. Constructing structural connectivity gradients}
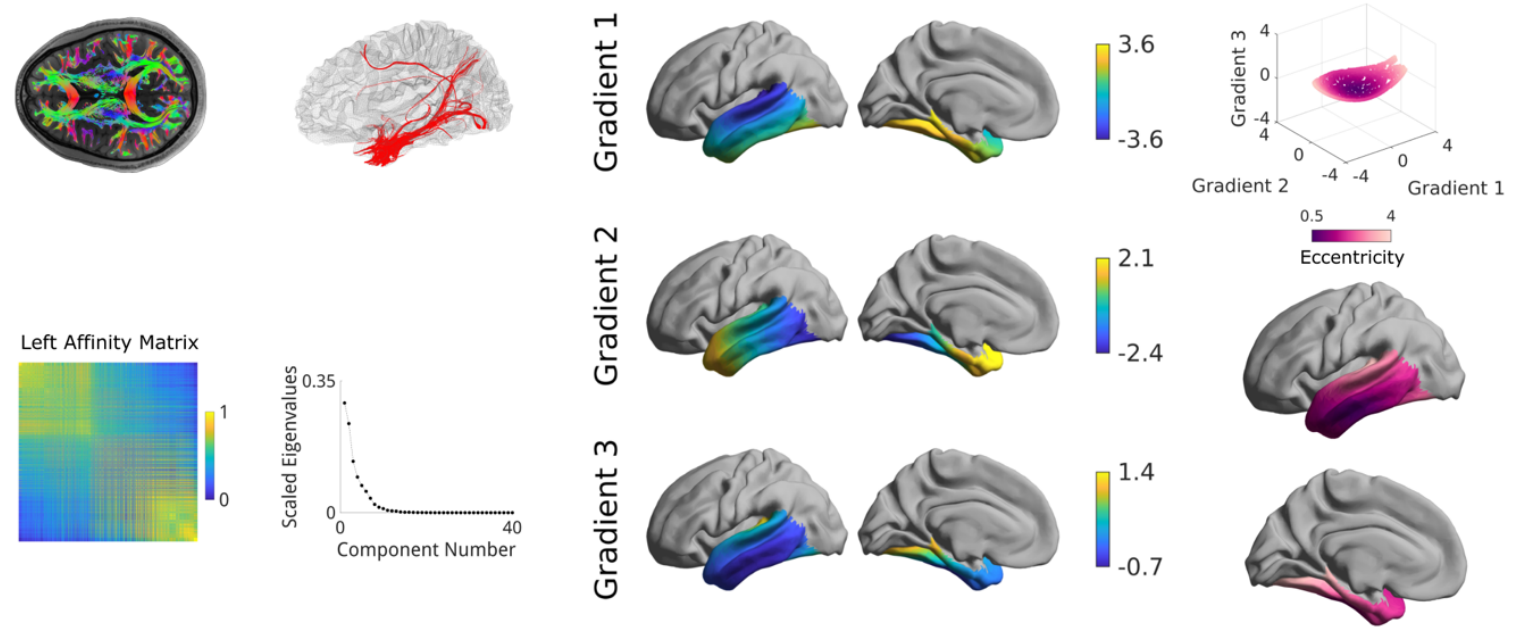

\section{B. Gradients represent connectivity distance}
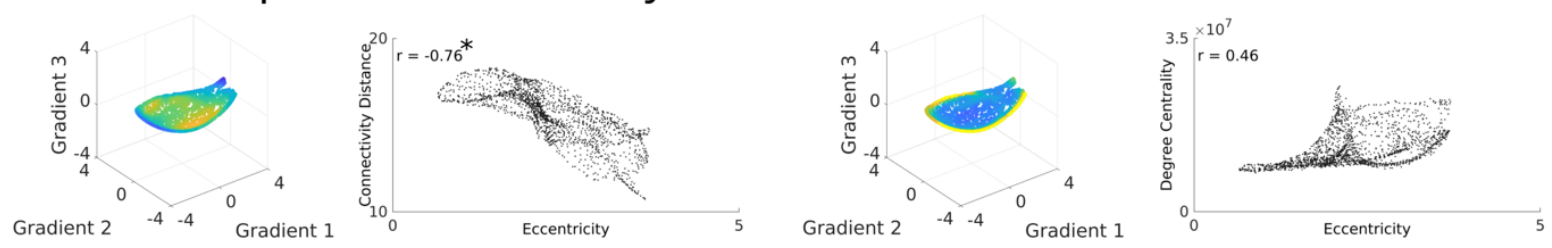

Fig. 1 Diffusion embedding of structural connectivity. (A) Streamlines were generated throughout the entire brain and systematically mapped to the cortical surface using nearest neighbor interpolation. We computed the affinity matrix of the connectivity matrix using a cosine similarity kernel and constructed gradients of structural connectivity of the temporal lobe to ipsilateral hemisphere with diffusion embedding. The first three gradients of diffusion embedding, sorted by variance explained of each vector, were selected for further analyses. Eccentricity in this manifold space was high in posterior medial temporal lobe and the superior temporal gyrus. (B) The relationship of eccentricity with connectivity distance (left) and degree centrality (right). The star denotes a significant correlation. 
In order to provide a scalar metric in multivariate gradient space and quantify connectome-level differentiation across the cortical mantle, we calculated an eccentricity measure that captures the distance from the origin in gradient space (Park, Bethlehem, et al., 2020). Low eccentricities were situated in the middle temporal gyrus, while high eccentricity was present in posterior superior temporal and medial temporal regions. To determine the connectivity patterns underlying gradient eccentricity, we performed spatial correlation analyses between eccentricity and topological measures of degree centrality and long-distance connectivity (Fig. 1B). Findings were corrected for spatial autocorrelation with Moran Spectral Randomization (Wagner \& Dray, 2015) implemented in BrainSpace (Vos de Wael et al., 2020), and adjusted for multiple comparisons using a false discovery rate procedure (Benjamini \& Hochberg, 1995). Gradient eccentricity correlated with connectivity distance in both hemispheres (left/right $\mathrm{r}=-0.76 /-0.70, \mathrm{p}_{\text {moran }}<0.002$ ), but not with degree centrality (left/right $\mathrm{r}=0.46 / 0.28$, $\mathrm{p}_{\text {moran }}<0.22$ ). Results replicated in all datasets i.e., gradients were bilaterally associated with connectivity distance (left/right HCP-Replication: $\mathrm{r}=-0.75 / 0.70, \mathrm{p}_{\text {moran }}<0.002 ;$ MICs: $\left.\mathrm{r}=-0.78 / 0.71, \mathrm{p}_{\mathrm{moran}}<0.01\right)$, but not degree centrality (HCPReplication: $\mathrm{r}=0.44 / 0.27, \mathrm{p}_{\operatorname{moran}}<0.23$, MICs: $\left.\mathrm{r}=0.40 / 0.39, \mathrm{p}_{\operatorname{moran}}<0.19\right)$.

To contextualize the gradients in their cognitive underpinnings, we decoded the structural gradients and eccentricity map using Neurosynth, an ad hoc meta-analysis of previous fMRI studies (Yarkoni et al., 2011) (Supplementary Fig. 3). Both G1, G3, and eccentricity represent axes of sensory functions to self-generated cognitive processes (G1: auditory vs memory/navigation terms, G3: cognitive vs auditory terms, eccentricity: cognitive vs perception terms). G2 differentiated stress/affect related terms from visual/word related terms (e.g., "visual" and "word form" vs "stress", "pain" and "regulation").

\section{Microstructural underpinnings}

Prior research in non-human animals has shown inter-regional connectivity is predicted by cytoarchitectural similarity (Barbas, 2015), and recent functional MRI work showed correspondence between functional gradients and proxies for intracortical myelin (Huntenburg et al., 2017; Larivière et al., 2019; Paquola et al., 2019; Vos de Wael et al., 2018). Here, we examined the relationship between structural connectivity gradients and in-vivo measures of cortical microstructure. Specifically, we tested for associations of gradient eccentricity and intracortical 
T1w/T2w intensity, a proxy for myelin (Glasser \& Essen, 2011), and observed a strong association (Fig. 2A; left/right $\mathrm{r}=0.69 / 0.78, \mathrm{p}_{\text {moran }}<0.012$ ). Associations to cortical thickness were only moderate $\left(\right.$ left/right $\mathrm{r}=-0.43 /-0.44$, $\left.\mathrm{p}_{\text {moran }}<0.024\right)$ and those to curvature did not reach statistical significance (left/right $\mathrm{r}=-0.07 /-0.11$, It $\mathrm{p}_{\text {moran }}<0.65$ ). Similar findings were seen in HCPReplication (left/right T1w/T2w r=0.68/0.78, $\mathrm{p}_{\text {moran }}<0.012$; cortical thickness $\mathrm{r}=-0.45 /$ 0.44, $\mathrm{p}_{\text {moran }}<0.020$; curvature $\mathrm{r}=-0.07 / 0.12, \mathrm{p}_{\text {moran }}<0.637$ ) and in the MICs dataset, which used quantitative T1 relaxometry as a myelin proxy (left/right $\mathrm{qT} 1 \mathrm{r}=-0.36 /-0.66 \mathrm{p}_{\text {moran }}<0.062$; cortical thickness $\mathrm{r}=-0.33 /-0.31, \mathrm{p}_{\text {moran }}<0.08$; curvature $\left.\mathrm{r}=0.01 / 0.03, \mathrm{p}_{\text {moran }}<0.91\right)$.

\section{A. Structural gradients and in-vivo cortical architecture}
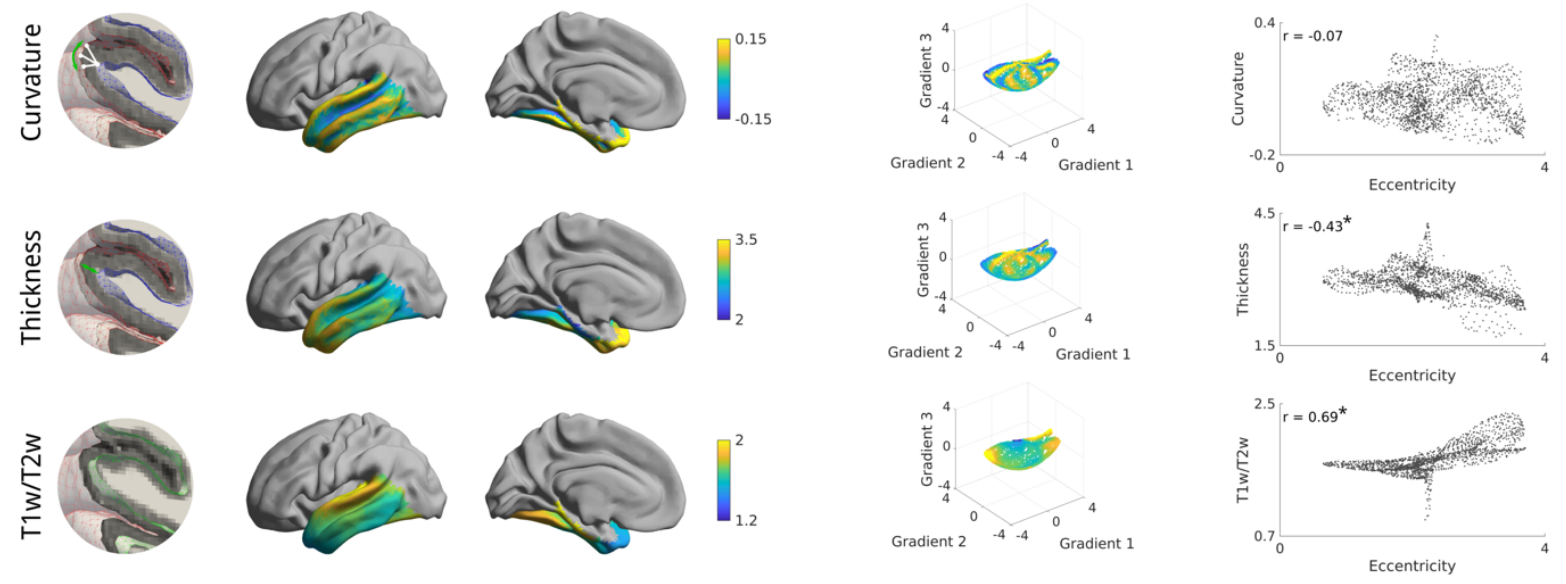

\section{B. Structural gradients and histology}
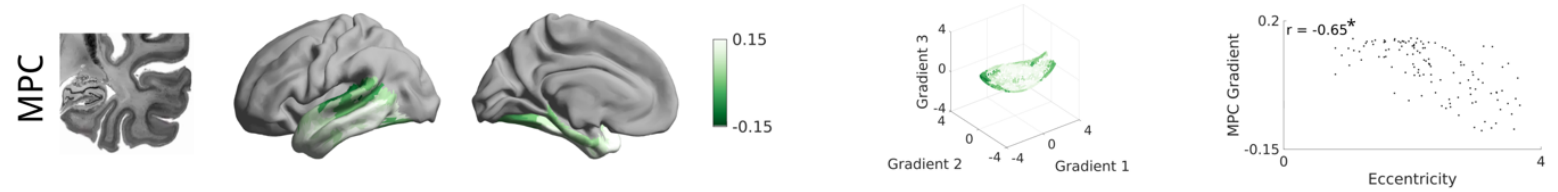

Fig. 2 Microstructural basis of the structural gradients. (A) We tested for linear relations between eccentricity and curvature, cortical thickness, as well as T1w/T2w intensity. Stars denote significant correlations. (B) We tested for an association between microstructural profile covariance derived previously from the BigBrain atlas (Amunts et al., 2013; Paquola et al., 2019), and eccentricity. Eccentricity was projected to the same parcellation scheme as microstructural profile covariance by taking the mean within each parcel.

Next, we evaluated the association between gradients and cortical cytoarchitecture (Error! Reference source not found.B), capitalizing on the BigBrain dataset, an ultra-high resolution 3D histological reconstruction of a post-mortem human brain (Amunts et al., 2013). We adopted a previously established approach to identify cytoarchitectural gradients (Paquola et al., 2019) and compared the principal cytoarchitectural gradient, which runs from primary sensory to limbic 
areas, to our in vivo structural connectivity gradients. We found strong associations in both hemispheres (left/right $\mathrm{r}=-0.65 /-0.73$, $\left.\mathrm{p}_{\text {moran }}<0.002\right)$. Again, results were replicated in both $H C P$ replication (left/right $\left.\mathrm{r}=-0.64 /-0.73, \quad \mathrm{p}_{\text {moran }}<0.002\right)$ and MICs (left/right $\mathrm{r}=-0.60 /-0.71$, $\left.\mathrm{p}_{\text {moran }}<0.028\right)$.

\section{Functional associations}

Structural connectivity is ultimately assumed to give rise to functional connectivity (Deco et al., 2017; Honey et al., 2009; Suárez et al., 2020; Wang et al., 2019). As such, we hypothesized that axes of structural connectivity would capture the organization of large-scale functional connectivity. We related the structural connectivity gradients to intrinsic functional community organization, a predominant motif of macroscale neural function (Fig. A) (Yeo et al., 2011). Using a 5-fold cross validation, we computed group-level structural gradients for the training and testing group. We derived beta values from the training sets with a group-level multinomial logistic regression and used those to predict the layout of the Yeo-Krienen intrinsic functional communities from the group-level testing set. Predictions were accurate and stable (Cohen's kappa mean + SD left/right: $0.77 \pm 0.01 / 0.81 \pm 0.01)$. Beta values derived from HCP-Discovery gradients also could accurately predict macroscale functional communities from the HCP-Replication (Cohen's kappa left/right: 0.77/ 0.82) as well as the MICs (Cohen's kappa left/right: 0.69/0.70).

To further assess the capacity of gradient features to predict regional functional connectivity, we leveraged decision tree regression with Euclidean distances between vertices in gradient space as predictors and edgewise functional connectivity within the temporal lobe as outcome variable. In a 5-fold cross validation trained on group-level folds of HCP-Discovery, gradient space distances were predictive of functional connectivity of held-out subjects at the single subject level (Fig. 3B; mean $\_$SD left/right: $\left.\mathrm{r}=0.50 \pm 0.04 / 0.46 \pm 0.05\right)$. A decision tree regression trained on the entire $H C P$-Discovery dataset accurately predicted single subject functional connectivity in both $H C P$ Replication (Fig. 3B; left/right $\mathrm{r}=0.49 \pm 0.05 / 0.43 \pm 0.05$ ) and MICs (left/right $\mathrm{r}=0.50 \pm 0.03$ / $\mathrm{r}=0.46 \pm 0.04)$. In all datasets prediction quality was excellent in the lateral temporal lobe but less favorable in the medial temporal lobe (Fig. 3C), possibly due to lower signal-to-noise ratio in this region. 


\section{A. Predicting Functional Networks}
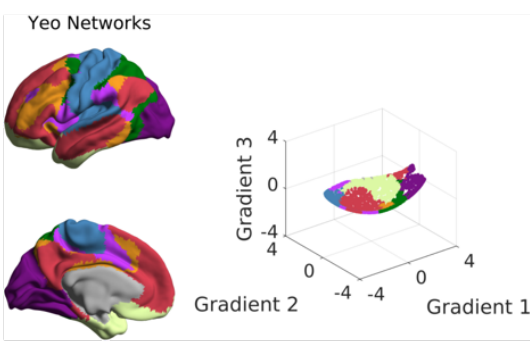

Predicted Networks
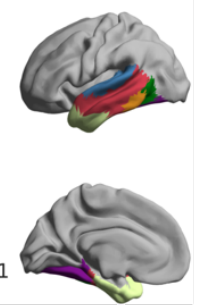

B. Decision Tree Regression: Subjectwise accuracy
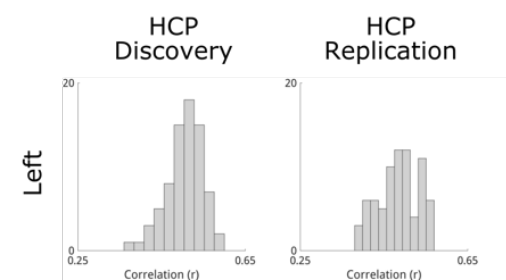

MICS
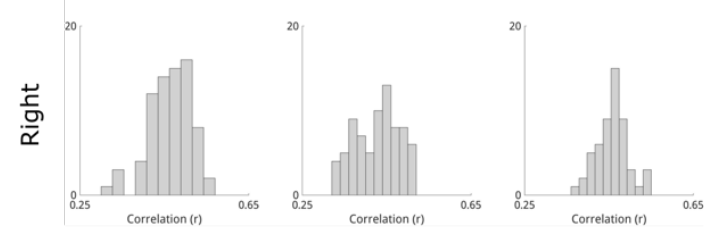

\section{Decision Tree Regression: Vertexwise accuracy}

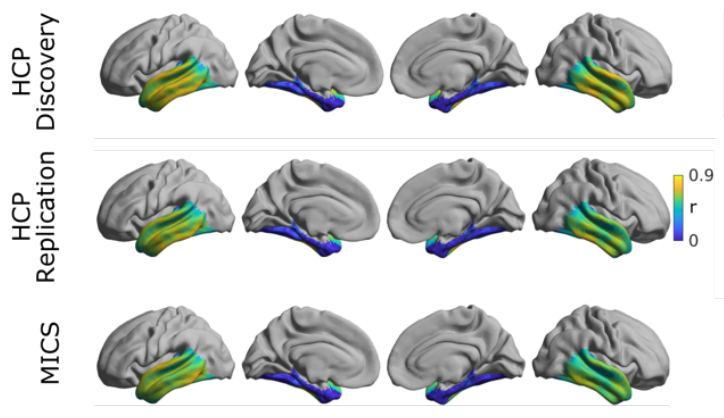

Fig. 3 Functional markers of the structural gradients. (A) Based on a canonical network parcellation (Yeo et al., 2011), we attempted to predict the functional networks. Performance was high with a Cohen's kappa of $0.77 \pm 0.01$ (left) and $0.81 \pm 0.01$ (right). Predicted networks shown here are the results of one of the five folds. (B) Histograms of the prediction accuracy per subject, as measured by the Pearson correlation between empirical and predicted data, of a decision tree regression estimating functional connectivity from structural gradients. HCPDiscovery predictions were trained with a 5-fold crossvalidation, the predictions of the other datasets were trained on HCP-Discovery (C) Pearson's correlation between the predicted and empirical functional connectivity for every vertex across subjects. Predictions were especially accurate in lateral temporal regions, and less robust in the medial temporal lobe.

\section{$\underline{\text { Evolutionary associations }}$}

Last, we assessed whether eccentricity also relates to measures of phylogenetic changes in the temporal lobe (Fig. 4). We found associations to previously established indices of functional homology (left/right: $\mathrm{r}=0.50 / 0.51, \mathrm{p}_{\text {moran }}<0.04$ ), a measure for similarity of functional organization between human and macaque, and areal expansion (left/right: $\mathrm{r}=-0.52 /-0.31, \mathrm{p}_{\text {moran }}<0.04$ ), a measure for the surface areas increase of human cortex relative to homologue regions in macaques (Xu et al., 2020). These results replicated both in $H C P$-Replication (functional homology; left/right: $\mathrm{r}=0.50 / 0.50, \mathrm{p}_{\operatorname{moran}}<0.04$;

areal expansion; left/right: $\mathrm{r}=-0.52 /-0.31$, $\mathrm{p}_{\text {moran }}<0.04$ ) as well as in $M I C s$ (functional homology; left/right: $\mathrm{r}=0.48 / 0.54, \mathrm{p}_{\text {moran }}<0.04$; areal expansion; left/right: $\mathrm{r}=-0.54 /-0.40, \mathrm{p}_{\text {moran }}<0.03$ ). 

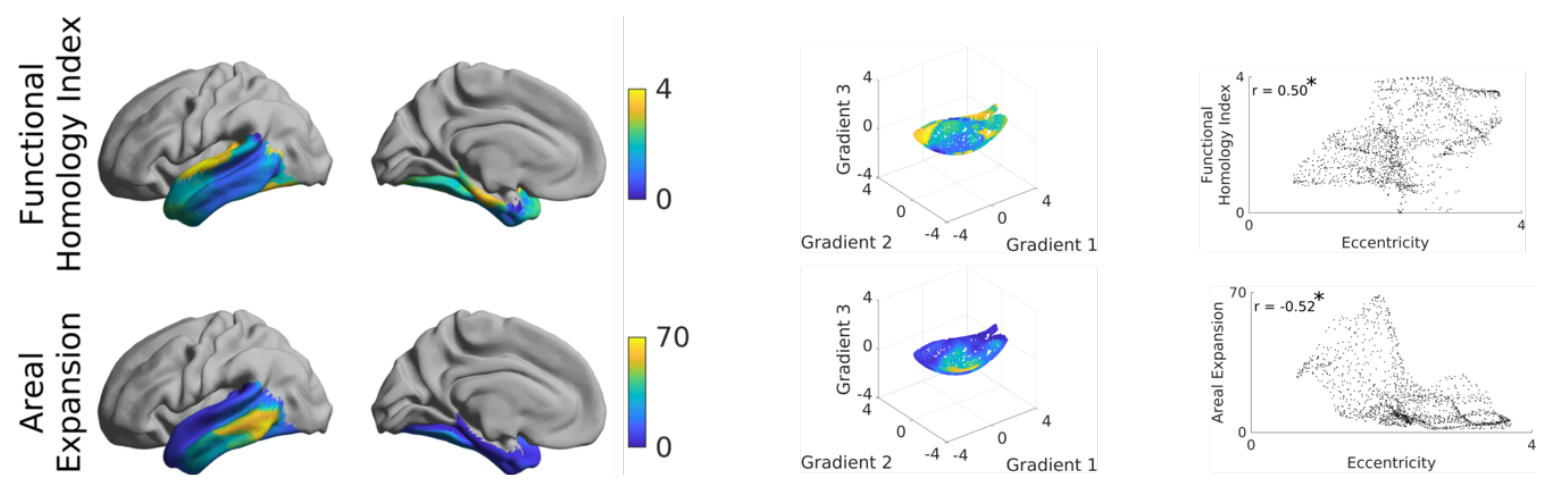

Fig 4. Relationship between the structural gradients and phylogenetic markers. Both functional homology and areal expansion are significantly associated with eccentricity of the first three structural gradients.

\section{DISCUSSION}

The temporal lobe hosts a diverse array of functional processes implicated in sensory processing, memory, and language abilities, and is among the macroscopic structures most frequently compromised in neurological and neuropsychiatric disorders, including Alzheimer's disease (Braak \& Braak, 1991) and drug-resistant epilepsy (Dam, 1982). To provide a comprehensive account of its substructural organization in humans, our study harnessed manifold learning operating on high resolution diffusion MRI tractography data of the temporal lobe to identify separate, yet partially overlapping axes of its structural connectome embedding. These axes were found to relate to MRI-based measures of intracortical myelination as well as post-mortem histology, supporting potential microcircuit underpinnings of these spatial trends in structural connectivity variations. Supervised learning experiments indicated that structural gradients can serve as sensitive, low-dimensional predictors of the functional organization of the temporal lobe. Notably, structural connectivity gradients also related to established markers of functional reconfiguration and areal expansion between humans and non-human primates, supporting the potential of connectome gradients in shaping evolutionary changes. Results were reproducible across multiple datasets, indicating generalizability. Collectively, our findings provide robust evidence for an association between tissue microstructure, structural connectivity, and functional motifs of the temporal lobe, which suggests their potential to serve as major organizational axis bridging between its microcircuit and macroscale layout.

Diffusion MRI is the only non-invasive method to approximate the course of white matter connections in humans. Based on multi-shell diffusion acquisitions of the $H C P$ and $M I C$ s datasets, 
we applied constrained spherical deconvolution (Jeurissen et al., 2014) and sphericaldeconvolution informed filtering of tractograms (R. E. Smith et al., 2015a) to estimate streamline weights interconnecting cortical areas. These techniques provide biologically meaningful weights of the modelled streamlines (R. E. Smith et al., 2015b), and reduce fiber tracking biases (Yeh et al., 2016) as well as partial volume effects (Jeurissen et al., 2014). By propagating each streamline to cortical surface points, rather than to macroscopic parcels, we were able to resolve fine grained changes in temporal lobe connectivity and thus account for heterogeneity of subregional connectivity. We enhanced this vertex-wise approach with manifold learning techniques that allow for the low-dimensional representation of spatial variations in temporal lobe structural connectivity. Already established by previous neuroimaging studies (Bajada et al., 2019; Blazquez Freches et al., 2020; Haak et al., 2018; Huntenburg et al., 2017; Margulies et al., 2016; Paquola et al., 2019), these techniques are able to model both gradual and overlapping modes of connectivity without reliance on a priori boundaries (Haak \& Beckmann, 2020). Recapitulating prior work, we found that the temporal lobe is best described by three gradients (Bajada et al., 2019; Blazquez Freches et al., 2020), spanning medio-lateral (G1), anterior-posterior (G2) and anterolateralposteromedial (G3) axes. Although there have been several reports of asymmetry of the white matter tracts of the temporal lobe, such as greater fiber density and tract volume in the left arcuate fasciculus than the right [for review see (Ocklenburg et al., 2016)], the symmetry of the structural gradients indicates gross similarity between large scale network embedding of left and right temporal lobes. We then tested for associations with MRI-based measures of curvature, cortical thickness, and intracortical microstructure. In line with our hypotheses and prior work suggesting a close link between internal cortical architecture and structural connectivity (Beul et al., 2017; García-Cabezas et al., 2019; Scannell et al., 1995; Young, 1992), we found strong associations between connectome gradients and MRI proxies of cortical myelin. The relationship with cortical thickness and curvature was weaker, suggesting that our cortical connectivity gradients closely reflect intracortical factors and only to a lesser extent macroscopic morphological variations and/or potential biases from sulco-gyral folding (Schilling et al., 2018). A closer link to microstructure was also suggested by harnessing BigBrain derived cytoarchitecture gradients (Amunts et al., 2013; Paquola et al., 2019). Collectively, these findings highlight the close relationship between microstructure and structural connectivity, supporting the extension of the structural model of 
connectivity to humans in the temporal lobes (Beul et al., 2017; García-Cabezas et al., 2019; Scannell et al., 1995; Young, 1992).

Many studies found that structural connectivity may predict functional connectivity by assuming that the strength of functional interactions depends, in part, on the density and efficacy of both direct and indirect structural connections (Deco et al., 2017; Honey et al., 2009; Wang et al., 2019). We hypothesized that the structural gradients, despite their low dimensionality, would still accurately describe functional interactions. Supervised learning approaches with cross-validation could show that gradient-informed models predicted the spatial layout of previously described intrinsic functional communities in the human brain (Yeo et al., 2011). At a more local scale, gradients could also predict patterns of inter-regional functional connectivity, even when trained and tested on datasets acquired from different scanners. Overall, our results support that lowdimensional eigenmode representations of structural connectivity may potentially underpin intrinsic functional architecture of the human connectome. Such a conclusion is in line with several prior studies in healthy individuals showing that whole-brain structural connectivity gradients shape dynamic signaling at rest (Park et al., 2021) as well as dynamic brain reconfigurations during tasks (C. Murphy et al., 2019). In the study of brain diseases associated with macroscale dysfunction, connectivity gradients have furthermore been used to contextualize changes in brain network architecture (Larivière et al., 2020; Li et al., 2020), supporting their utility to serve as coordinate systems of macroscale functional interactions in healthy and diseased brains.

Cross-species comparisons provide a potential window into human uniqueness by studying brain reconfigurations between humans and non-human primates (Buckner \& Krienen, 2013; Krubitzer, 2007). Whilst a remarkable conservation of macroscale organizational principles between macaques and humans is evident (Glasser et al., 2014; Margulies et al., 2016; Valk et al., 2020), association cortices have specifically undergone a striking expansion in relative surface and potential participation in distributed functional networks (Buckner \& Krienen, 2013; Hill et al., 2010; Mars et al., 2017; Mueller et al., 2013; Patel et al., 2015). Here, we showed that structural connectivity gradients spatially align with the pattern of evolutionarily diverging brain areas and areal expansion, an index for relative areal size differences across species (Xu et al., 2020). Areas near the center of the structural manifold were less functionally homologous and have undergone more expansion relative to macaques. This may indicate that evolutionary changes have preferentially occurred along particular fiber tracts including, for example, the arcuate fasciculus 
which has undergone critical anatomical modifications between non-human and human primates (Ardesch et al., 2019; Eichert et al., 2019, 2020; Mars, Eichert, et al., 2018; Mars et al., 2013; Rilling et al., 2008). When taken together with the cognitive terms from the Neurosynth metaanalysis, these results indicate that phylogenetic differences in the temporal lobe are primarily situated along those tracts associated with self-generated cognitive processes.

Theoretical accounts, empirical findings, and gradual changes in research culture have increased the scientific value of replications in neuroscience (Ioannidis, 2005; Moonesinghe et al., 2007; Open Science Collaboration, 2015). Here, we replicated our findings in two datasets: 1) a set of unrelated young adults derived from the same dataset as the discovery set (HCP-Replication) as well as 2) a separate dataset acquired at the Montreal Neurological Institute (MICs). Even after stringent corrections for both spatial autocorrelation (Wagner \& Dray, 2015) and multiple comparisons (Benjamini \& Hochberg, 1995), most findings held across all datasets indicating good reproducibility. We have released all utilized feature data and associated analysis scripts (https://github.com/MICA-MNI/micaopen), for independent verification of our results and followup analysis. We hope that these data and associated findings continue to pave the way into studying the important relationship between the microstructure, connectivity, and evolutionary development of the temporal lobe.

\section{METHODS}

\section{Participants}

We selected 150 unrelated participants from the Human Connectome Project dataset for whom all resting-state, diffusion weighted imaging and structural scans were available and completed in full (Van Essen et al., 2013). These participants were split into HCP-Discovery $(\mathrm{n}=75$; age $=29.2 \pm 3.6$, female $=47)$ and HCP-Replication $(\mathrm{n}=75$; age $=28.9 \pm 4.0$, female=44) datasets. For the MICs dataset, all data were collected in a single testing session per participant between April 2018 and March 2020. Participants $(n=54 ; 30.5 \pm 7.3$, female=20) all provided informed consent. Participants reported no history of neurological illness. The study was approved by the Ethics Committee of the Montreal Neurological Institute and Hospital. 


\section{Image Acquisition}

a) HCP. Images were acquired on the customized Siemens 3T "Connectome Skyra". Two T1w images were acquired with a 3D MPRAGE sequence with the following parameters: $\mathrm{TR}=2400 \mathrm{~ms}$, $\mathrm{TE}=2.14 \mathrm{~ms}$, flip angle $=8 \mathrm{deg}, \mathrm{FOV}=224 \times 224 \mathrm{~mm}^{2}$, voxel size $=0.7 \mathrm{~mm}$ isotropic. Two T2w images were acquired with identical parameters except for the following: $\mathrm{TR}=3200 \mathrm{~ms}, \mathrm{TE}=565 \mathrm{~ms}$, variable flip angle. Four resting-state fMRI images were acquired with a gradient-echo echo-planar imaging (EPI) sequence $(\mathrm{TR}=720 \mathrm{~ms}, \mathrm{TE}=33.1 \mathrm{~ms}$, flip angle=52 deg, FOV=208x180 mm, $2 \mathrm{~mm}$ isotropic voxels, and 1200 volumes per run). Diffusion images were acquired with a spin-echo EPI sequence $(\mathrm{TR}=5520 \mathrm{~ms}, \mathrm{TE}=89.5 \mathrm{~ms}$, flip angle $=78 \mathrm{deg}, \mathrm{FOV}=210 \times 180 \mathrm{~mm}, 1.25 \mathrm{~mm}$ isotropic voxels, b-values 1000, 2000, and $3000 \mathrm{~s} / \mathrm{mm}^{2}$, 90 diffusion weighting directions). Six diffusion image scans were acquired each lasting 9 minutes and 50 seconds. Half the runs were acquired with left-to-right phase encoding and the other half with right-to-left.

b) MICs. Images were acquired on a 3T Siemens Magnetom Prisma-Fit equipped with a 64channel head coil. Two T1w scans were acquired with a 3D-MPRAGE sequence $(0.8 \mathrm{~mm}$ isotropic voxels, matrix $=320 \times 320,224$ sagittal slices, $\mathrm{TR}=2300 \mathrm{~ms}, \mathrm{TE}=3.14 \mathrm{~ms}$, $\mathrm{TI}=900 \mathrm{~ms}$, flip angle $=9^{\circ}$, iPAT=2). Quantitative T1 (qT1) relaxometry data was acquired using a 3D-MP2RAGE sequence $(0.8 \mathrm{~mm}$ isotropic voxels, 240 sagittal slices, TR $=5000 \mathrm{~ms}, \mathrm{TE}=2.9 \mathrm{~ms}, \mathrm{TI} 1=940 \mathrm{~ms}, \mathrm{~T} 12=2830 \mathrm{~ms}$, flip angle $1=4^{\circ}$, flip angle $2=5^{\circ}, \mathrm{iPAT}=3$, bandwidth $=270 \mathrm{~Hz} / \mathrm{px}$, echo spacing $=7.2 \mathrm{~ms}$, partial Fourier=6/8). We combined two inversion images for qT1 mapping to minimise sensitivity to B1 inhomogeneities and optimize intra- and inter-subject reliability (Haast et al., 2016; Marques et al., 2010). DWI images were obtain with spin-echo EPI, including three shells with b-values 300, 700 , and $2000 \mathrm{~s} / \mathrm{mm}^{2}$ and 10,40 , and 90 diffusion weighting directions per shell, respectively $\left(\mathrm{TR}=3500 \mathrm{~ms}, \mathrm{TE}=64.40 \mathrm{~ms}, 1.6 \mathrm{~mm}\right.$ isotropic voxels, flip angle $=90^{\circ}$, refocusing flip angle $=180^{\circ}$, FOV $=224 \times 224 \mathrm{~mm}^{2}$, slice thickness $=1.6 \mathrm{~mm}$, multiband factor $=3$, echo spacing $=0.76 \mathrm{~ms}$, number of b0 images=3). One 7 min rs-fMRI scan was acquired using multiband accelerated 2D-BOLD EPI $\left(\mathrm{TR}=600 \mathrm{~ms}, \mathrm{TE}=30 \mathrm{~ms}, 3 \mathrm{~mm}\right.$ isotropic voxels, flip angle $=52^{\circ}, \mathrm{FOV}=240 \times 240 \mathrm{~mm}^{2}$, slice thickness $=3 \mathrm{~mm}$, multiband factor $=6$, echo spacing $=0.54 \mathrm{~ms}$ ). Participants were instructed to keep their eyes open, look at a fixation cross, and not fall asleep. Two spin-echo images with reverse phase encoding were acquired for distortion correction of the rsfMRI scans (phase encoding $=\mathrm{AP} / \mathrm{PA}, \quad 3 \mathrm{~mm}$ isotropic voxels, $\mathrm{FOV}=240 \times 240 \mathrm{~mm}^{2}$, slice thickness $=3 \mathrm{~mm}$, $\mathrm{TR}=4029 \mathrm{~ms}, \mathrm{TE}=48 \mathrm{~ms}$, flip angle $=90^{\circ}$, echo spacing $=0.54 \mathrm{~ms}$, bandwidth $\left.=2084 \mathrm{~Hz} / \mathrm{Px}\right)$. 


\section{Structural Preprocessing}

a) HCP. Structural images underwent standard HCP preprocessing (Glasser et al., 2013). In short, T1w images were corrected for gradient nonlinearity. Repeated scans were co-registered and averaged. After brain extraction and readout distortion correction, T1w and T2w images were coregistered using rigid body transformations. Non-uniformity correction based on the T1w and T2w contrasts was applied. Preprocessed images were nonlinearly registered to MNI152 space. Cortical surfaces were extracted using FreeSurfer 5.3.0-HCP (Dale et al., 1999; Fischl, Sereno, \& Dale, 1999; Fischl, Sereno, Tootell, et al., 1999), with minor modifications to incorporate information from both T1w and T2w scans. Cortical surfaces were aligned using MSMAll to the Conte69 template (Robinson et al., 2014).

b) MICs. Data were preprocessed with a Freesurfer 6.0 recon_all pipeline. Both native T1w scans were provided as input and combined through this pipeline. Manual corrections of the pial and white matter surfaces were performed for all subjects. Curvature and cortical thickness estimates were generated by the recon_all pipeline. To acquire tissue segmentations for anatomically constrained tractography, the same images underwent a separate pipeline which included linear alignment of both $\mathrm{T} 1 \mathrm{w}$ scans, non-uniformity correction, and intensity normalization. Corrected images were segmented into tissue types using MRtrix3's 5ttgen (R. E. Smith et al., 2012). qT1 images were linearly aligned to the cortical surface using boundary based registration (Greve \& Fischl, 2009). qT1 values were interpolated to the surface by taking the average of seven trilinear interpolations evenly interspersed between the $20^{\text {th }}$ and $80^{\text {th }}$ percentile distances from the pial to white matter surfaces using Freesurfer's mri_vol2surf command.

\section{Resting-State Preprocessing}

a) HCP. Data underwent standard HCP preprocessing (Glasser et al., 2013). In short, the timeseries were corrected for gradient non-linearity and head-motion. The R-L/L-R scan pairs we used to correct for geometric distortions. Resulting images were warped to the structural image using rigid body and boundary-based registrations. This warp was concatenated with the warp from $\mathrm{T} 1 \mathrm{w}$ image space to MNI152 space to transform functional images to MNI152 space. Further processing removed the bias field, removed the skull, and normalized whole brain intensity. A high pass filter ( $>2000$ s FWHM) was used to correct for scanner drift, and additional noise was removed using ICA-FIX (Salimi-Khorshidi et al., 2014). 
b) MICs. The first five volumes were discarded to allow for magnetic field saturation. Images were then reoriented, and motion and distortion corrected. Nuisance variable signal was removed using an ICA-FIX classifier trained on this dataset and subsequent spike regression (Salimi-Khorshidi et al., 2014). Further tissue-specific signal regression was not performed (K. Murphy \& Fox, 2017; Vos de Wael et al., 2017). A warp to the Freesurfer T1w image was computed by averaging volumetric timeseries across the time dimension and registering this image using boundary-based registration. Timeseries were sampled to the surface by taking the average of seven trilinear interpolations evenly interspersed between the $20^{\text {th }}$ and $80^{\text {th }}$ percentile distances from the pial to white matter surfaces.

\section{Diffusion Preprocessing}

a) HCP. Images underwent standard HCP preprocessing (Glasser et al., 2013). In short, image intensity was normalized across scans. The topup and eddy algorithms were used to correct for EPI distortions, eddy currents, and motion. A gradient nonlinearity correction was performed, and the deviation of the b-values and b-vectors was computed. Mean b0 images were registered to the T1w image with boundary-based registration (Greve \& Fischl, 2009), and this registration was used to transform DWI images to T1w space. The brain was masked based on a Freesurfer segmentation.

b) MICs. Data were preprocessed and denoised with MRTrix3's dwipreproc, which is based on FSL's eddy correction and topup, and dwidenoise (Andersson et al., 2003; S. M. Smith et al., 2004; Tournier et al., 2012). Freesurfer segmentations were registered to the subject's DWI space using boundary-based registration (Greve \& Fischl, 2009).

\section{High resolution diffusion tractography and gradient mapping}

Tractography was performed identically for the HCP and MICs dataset with MrTrix3 (Tournier et al., 2012). Response functions for each tissue type were estimated using the dhollander algorithm (Dhollander et al., 2016). Fiber orientation distributions were modelled with multi-shell multitissue spherical deconvolution (Jeurissen et al., 2014) and subsequently underwent multi-tissue informed log-domain intensity normalization. The structural $\mathrm{T} 1 \mathrm{w}$ image was segmented into five tissue types (R. E. Smith et al., 2012). Anatomically constrained tractography was performed systematically for each temporal lobe voxel in the gray-white matter interface by generating streamlines using second order integration over fiber orientation distributions (Tournier et al., 
2010). Streamlines were seeded dynamically from the white matter using the SIFT model (R. E. Smith et al., 2015a). Streamline generation was aborted when 40 million streamlines had been accepted. Each streamline was assigned a weight by optimizing a cross-section multiplier derived with the SIFT2 algorithm (R. E. Smith et al., 2015a). Streamline termini were assigned to their nearest vertex on the surface of the gray-white matter interface. Streamlines of which either terminus was further than $3 \mathrm{~mm}$ from its nearest vertex were discarded. Connectomes were smoothed on the surface using a $20 \mathrm{~mm}$ full width at half maximum Gaussian smoothing kernel.

To describe the largest axes of variance in connectivity we used diffusion map embedding (Coifman \& Lafon, 2006), a non-linear dimensionality reduction techniques technique used previously to identify neocortical, hippocampal, and cerebellar functional gradients (Guell et al., 2018; Margulies et al., 2016; Vos de Wael et al., 2018). Gradients were computed and aligned using the BrainSpace toolbox (https:/github.com/MICA-MNI/BrainSpace) (Vos de Wael et al., 2020), with the following settings: sparsity thresholding at the $75^{\text {th }}$ percentile, a cosine affinity kernel, diffusion map embedding dimensionality reduction with $\mathrm{a}=0.5$, and automated diffusion time estimation. Gradient computations were performed separately on left and right temporal lobes. Interhemispheric connections were not included in the gradient computation. Left and right gradients were aligned with Procrustes alignment (Langs et al., 2015) as implemented in BrainSpace. Eccentricity was computed from the aligned gradients by computing the Euclidean distance to the origin of the manifold space spanned by the first three gradients.

\section{Statistical testing}

Testing for linear associations between cortical markers and gradients likely leads to biased test statistics due to the spatial autocorrelation of MRI data violating the independence of observations assumption (Alexander-Bloch et al., 2018). Instead, for each statistical test we generated random datasets with comparable spatial properties. Specifically, we generated random datasets with equivalent spatial autocorrelation as the response variable using Moran spectral randomization with the singleton procedure (Wagner \& Dray, 2015) as implemented in BrainSpace (Vos de Wael et al., 2020). All linear models were fitted for the original data as well as 1000 corresponding simulated datasets. Presented p-values were derived from the percentile rank of the true F-statistic in the distribution of F-statistics in the simulated data. Multiple comparison were corrected for false discovery rate with the Benjamini-Hochberg procedure (Benjamini \& Hochberg, 1995). 


\section{Tractography analyses}

Connectivity distance, a measure that characterizes the relationship between physical distance and connectivity (Larivière et al., 2020), was computed by thresholding the structural connectivity matrix at the $80^{\text {th }}$ percentile, multiplying each connection by the geodesic distance between their nodes, and averaging all connections for each node. Degree centrality was computed as the column-wise sum of the connectivity matrix. Statistical significance of the association between both degree centrality as well as connectivity distance with the gradients was assessed with Moran spectral randomization (Wagner \& Dray, 2015).

\section{BigBrain Gradient}

To assess histological properties of the brain we used BigBrain, an ultrahigh resolution atlas of a single post-mortem brain stained for cell bodies (Amunts et al., 2013). Gradients of microstructural profile covariance were computed as described previously (Paquola et al., 2019). In short, 18 equivolumetric surfaces were constructed between the outer and inner cortical surfaces. To reduce partial volume effects, the inner cortical surface was removed. A linear model implemented in SurfStat (Worsley et al., 2009) was used to correct for anterior-to-posterior increases in intensity values (Amunts et al., 2013). Surface vertices were grouped into 1012 parcels which respected the boundaries of the Desikan-Killiany atlas (Desikan et al., 2006; Hong et al., 2017). A microstructural profile covariance matrix was constructed by computing the Pearson correlation of every pair of vectors whilst controlling for the average whole-cortex intensity profile. Gradients were constructed from this matrix using BrainSpace default parameters ( $90 \%$ sparsity, normalized angle kernel, diffusion map embedding, $\mathrm{a}=0.5$, automated diffusion time estimation). To compare structural connectivity gradients to BigBrain gradients, the structural gradients were parcellated using the same parcellation scheme. Moran spectral randomization (Wagner \& Dray, 2015) was used to test for associations between BigBrain gradient 1 and the structural gradients.

\section{Functional Predictions}

Structural gradients were used to predict canonical resting-state networks published previously [(Yeo et al., 2011); https://surfer.nmr.mgh.harvard.edu/fswiki/CorticalParcellation_Yeo2011]. HCP-Discovery was split into five folds of 15 subjects each; for each fold we performed a multinomial logistic regression with the first three gradients as predictor variables and networks as outcome variables. Beta values derived from the training set were used to predict probabilities 
of each network in the testing set. Each vertex was assigned to the network with the highest probability. Additionally, we derived beta values from the entire HCP-Discovery dataset and used these to predict functional networks from HCP-Replication and MICs gradients.

To further assess the relationship between structural gradients and edgewise functional connectivity, we used a decision tree with binary splits for regression. Similar to the network prediction, training and testing was performed both with 5-fold cross validation as well as training on HCP-Discovery and testing on the other datasets. Model training was performed with the fitrtree function as implemented in MATLAB R2019b with a minimum leaf size of 20, a maximum number of splits of 20 , and otherwise default parameters.

\section{Evolutionary Analyses}

We tested for associations between our gradients and two markers of evolutionary change between humans and macaques: functional homology and areal expansion. Both measures were presented in a prior paper (Xu et al., 2020), hence we only provide a short overview here. Functional homology is a measure for the functional similarity of a human brain area with its macaque counterpart. It is computed based on the maximum cosine similarity of functional gradient profiles within a $12 \mathrm{~mm}$ search light around the corresponding human/macaque vertices. An areal expansion map shows the relative expansion of human cortex compared to macaques. It is computed by dividing the local area of human cortex by the corresponding area of macaque cortex where correspondence was defined based on functional homology. We tested for associations between these two markers and the structural gradients using Moran spectral randomization (Wagner \& Dray, 2015).

\section{ACKNOWLEDGEMENTS}

Reinder Vos de Wael was funded by studentships from the Savoy foundation for Epilepsy and the Richard and Ann Sievers award. Dr. Jessica Royer was supported by a fellowship from the Canadian Open Neuroscience Platform (CONP) and CIHR. Shahin Tavakol was funded by a studentship from McGill University's Faculty of Medicine. Dr Casey Paquola was funded through a postdoctoral fellowship of the Fonds de la Recherche du Quebec - Santé (FRQ-S). Dr Oualid Benkarim was funded by a Healthy Brains for Healthy Lives (HBHL) postdoctoral fellowship. Sara Larivière was funded by the Canadian Institutes of Health Research (CIHR). Dr Jonathan 
Smallwood was supported by the European Research Council (WANDERINGMINDSERC646927). Dr Boris Bernhardt acknowledges research support from the National Science and Engineering Research Council of Canada (NSERC Discovery-1304413), the Canadian Institutes of Health Research (CIHR FDN-154298), SickKids Foundation (NI17-039), Azrieli Center for Autism Research (ACAR-TACC), and the Tier-2 Canada Research Chairs program.

Data were provided, in part, by the Human Connectome Project, WU-Minn Consortium (Principal Investigators: David Van Essen and Kamil Ugurbil; 1U54MH091657) funded by the 16 NIH Institutes and Centers that support the NIH Blueprint for Neuroscience Research; and by the McDonnell Center for Systems Neuroscience at Washington University.

\section{COMPETING INTERESTS}

No author declares competing interests. 


\section{References}

Alexander-Bloch, A. F., Shou, H., Liu, S., Satterthwaite, T. D., Glahn, D. C., Shinohara, R. T., Vandekar, S. N., \& Raznahan, A. (2018). On testing for spatial correspondence between maps of human brain structure and function. NeuroImage. https://doi.org/10.1016/j.neuroimage.2018.05.070

Amunts, K., Lepage, C., Borgeat, L., Mohlberg, H., Dickscheid, T., Rousseau, M.-É., Bludau, S., Bazin, P.-L., Lewis, L. B., Oros-Peusquens, A.-M., \& others. (2013). BigBrain: An ultrahigh-resolution 3D human brain model. Science, 340(6139), 1472-1475.

Andersson, J. L., Skare, S., \& Ashburner, J. (2003). How to correct susceptibility distortions in spin-echo echo-planar images: Application to diffusion tensor imaging. NeuroImage, $20(2), 870-888$.

Ardesch, D. J., Scholtens, L. H., Li, L., Preuss, T. M., Rilling, J. K., \& Heuvel, M. P. van den. (2019). Evolutionary expansion of connectivity between multimodal association areas in the human brain compared with chimpanzees. Proceedings of the National Academy of Sciences, 116(14), 7101-7106. https://doi.org/10.1073/pnas.1818512116

Attar, F. M., Kirilina, E., Haenelt, D., Pine, K., Trampel, R., Edwards, L., \& Weiskopf, N. (2020). Mapping short association fibers in the early cortical visual processing stream using in vivo diffusion tractography. Cerebral Cortex, 30(8), 4496-4514. https://doi.org/10.1093/cercor/bhaa049

Bajada, C. J., Jackson, R. L., Haroon, H. A., Azadbakht, H., Parker, G. J. M., Lambon Ralph, M. A., \& Cloutman, L. L. (2017). A graded tractographic parcellation of the temporal lobe. NeuroImage, 155, 503-512. https://doi.org/10.1016/J.NEUROIMAGE.2017.04.016

Bajada, C. J., Trujillo-Barreto, N. J., Parker, G. J. M., Cloutman, L. L., \& Lambon Ralph, M. A. (2019). A structural connectivity convergence zone in the ventral and anterior temporal 
lobes: Data-driven evidence from structural imaging. Cortex, 120, 298-307. https://doi.org/10.1016/j.cortex.2019.06.014

Ball, G., Seidlitz, J., Beare, R., \& Seal, M. L. (2020). Cortical remodelling in childhood is associated with genes enriched for neurodevelopmental disorders. NeuroImage, 215, 116803. https://doi.org/10.1016/j.neuroimage.2020.116803

Ball, G., Seidlitz, J., O’Muircheartaigh, J., Dimitrova, R., Fenchel, D., Makropoulos, A., Christiaens, D., Schuh, A., Passerat-Palmbach, J., Hutter, J., Cordero-Grande, L., Hughes, E., Price, A., Hajnal, J. V., Rueckert, D., Robinson, E. C., \& Edwards, A. D. (2020). Cortical morphology at birth reflects spatio-temporal patterns of gene expression in the fetal human brain. BioRxiv. https://doi.org/10.1101/2020.01.28.922849

Barbas, H. (2015). General Cortical and Special Prefrontal Connections: Principles from Structure to Function. Annual Review of Neuroscience, 38(1), 269-289. https://doi.org/10.1146/annurev-neuro-071714-033936

Benjamini, Y., \& Hochberg, Y. (1995). Controlling the False Discovery Rate. J. Royal Stat. Soc. $B, 57,2890399$.

Bethlehem, R. A. I., Paquola, C., Seidlitz, J., Ronan, L., Bernhardt, B., Consortium, C.-C., \& Tsvetanov, K. A. (2020). Dispersion of functional gradients across the lifespan. BioRxiv, 2020.02.27.968537. https://doi.org/10.1101/2020.02.27.968537

Beul, S. F., Barbas, H., \& Hilgetag, C. C. (2017). A Predictive Structural Model of the Primate Connectome. Scientific Reports, 7, 43176. https://doi.org/10.1038/srep43176

Bijsterbosch, J., Harrison, S. J., Jbabdi, S., Woolrich, M., Beckmann, C., Smith, S., \& Duff, E. P. (2020). Challenges and future directions for representations of functional brain 
organization. Nature Neuroscience, 23(12), 1484-1495. https://doi.org/10.1038/s41593020-00726-Z

Blazquez Freches, G., Haak, K. V., Bryant, K. L., Schurz, M., Beckmann, C. F., \& Mars, R. B. (2020). Principles of temporal association cortex organisation as revealed by connectivity gradients. Brain Structure and Function, 225(4), 1245-1260. https://doi.org/10.1007/s00429-020-02047-0

Bodin, C., Pron, A., Mao, M. L., Régis, J., Belin, P., \& Coulon, O. (2020). Plis de passage in the Superior Temporal Sulcus: Morphology and local connectivity. BioRxiv, 2020.05.26.116152. https://doi.org/10.1101/2020.05.26.116152

Bonilha, L., Hillis, A. E., Hickok, G., den Ouden, D. B., Rorden, C., \& Fridriksson, J. (2017). Temporal lobe networks supporting the comprehension of spoken words. Brain, 140(9), 2370-2380. https://doi.org/10.1093/brain/awx169

Braak, H., \& Braak, E. (1991). Neuropathological stageing of Alzheimer-related changes. Acta Neuropathologica, 82(4), 239-259.

Bryant, K. L., Li, L., Eichert, N., \& Mars, R. B. (2020). A comprehensive atlas of white matter tracts in the chimpanzee. PLOS Biology, In Press. http://biorxiv.org/content/early/2020/01/25/2020.01.24.918516.abstract

Buckner, R. L., \& Krienen, F. M. (2013). The evolution of distributed association networks in the human brain. Trends in Cognitive Sciences, 17(12), 648-665.

Buckner, R. L., \& Margulies, D. (2019). Macroscale cortical organization and a default-like apex transmodal network in the marmoset monkey. Nat Commun, 10, 1976.

Coifman, R. R., \& Lafon, S. (2006). Diffusion maps. Applied and Computational Harmonic Analysis, 21(1), 5-30. https://doi.org/10.1016/j.acha.2006.04.006 
Dale, A. M., Fischl, B., \& Sereno, M. I. (1999). Cortical surface-based analysis: I. Segmentation and surface reconstruction. NeuroImage, $9(2), \quad 179-194$. https://doi.org/10.1006/nimg.1998.0395

Dam, A. M. (1982). Hippocampal neuron loss in epilepsy and after experimental seizures. Acta Neurologica Scandinavica, 66(6), 601-642. https://doi.org/10.1111/j.16000404.1982.tb04528.x

Deco, G., Kringelbach, M. L., Jirsa, V. K., \& Ritter, P. (2017). The dynamics of resting fluctuations in the brain: Metastability and its dynamical cortical core. Scientific Reports, 7(1), 3095. https://doi.org/10.1038/s41598-017-03073-5

Desikan, R. S., Ségonne, F., Fischl, B., Quinn, B. T., Dickerson, B. C., Blacker, D., Buckner, R. L., Dale, A. M., Maguire, R. P., Hyman, B. T., \& others. (2006). An automated labeling system for subdividing the human cerebral cortex on MRI scans into gyral based regions of interest. Neuroimage, 31(3), 968-980.

Dhollander, T., Raffelt, D., \& Connelly, A. (2016). Unsupervised 3-tissue response function estimation from single-shell or multi-shell diffusion MR data without a co-registered T1 image. ISMRM Workshop on Breaking the Barriers of Diffusion MRI.

Eichert, N., Robinson, E. C., Bryant, K. L., Jbabdi, S., Jenkinson, M., Li, L., Krug, K., Watkins, K. E., \& Mars, R. B. (2020). Cross-species cortical alignment identifies different types of anatomical reorganization in the primate temporal lobe. ELife, 9, e53232. https://doi.org/10.7554/eLife.53232

Eichert, N., Verhagen, L., Folloni, D., Jbabdi, S., Khrapitchev, A. A., Sibson, N. R., Mantini, D., Sallet, J., \& Mars, R. B. (2019). What is special about the human arcuate fasciculus? 
Lateralization, projections, and expansion. Cortex, 118, 107-115. https://doi.org/10.1016/j.cortex.2018.05.005

Fischl, B., Sereno, M. I., \& Dale, A. M. (1999). Cortical surface-based analysis: II. Inflation, flattening, and a surface-based coordinate system. NeuroImage, 9(2), 195-207. https://doi.org/10.1006/nimg.1998.0396

Fischl, B., Sereno, M. I., Tootell, R. B. H., \& Dale, A. M. (1999). High-resolution intersubject averaging and a coordinate system for the cortical surface. Human Brain Mapping.

García-Cabezas, M. Á., Zikopoulos, B., \& Barbas, H. (2019). The Structural Model: A theory linking connections, plasticity, pathology, development and evolution of the cerebral cortex. Brain Structure and Function, 224(3), 985-1008. https://doi.org/10.1007/s00429019-01841-9

Glasser, M. F., \& Essen, D. C. V. (2011). Mapping Human Cortical Areas In Vivo Based on Myelin Content as Revealed by T1- and T2-Weighted MRI. Journal of Neuroscience, 31(32), 11597-11616. https://doi.org/10.1523/JNEUROSCI.2180-11.2011

Glasser, M. F., Goyal, M. S., Preuss, T. M., Raichle, M. E., \& Van Essen, D. C. (2014). Trends and Properties of Human Cerebral Cortex: Correlations with Cortical Myelin Content. NeuroImage, 93 Pt 2, 165-175. https://doi.org/10.1016/j.neuroimage.2013.03.060

Glasser, M. F., Sotiropoulos, S. N., Wilson, J. A., Coalson, T. S., Fischl, B., Andersson, J. L., Xu, J., Jbabdi, S., Webster, M., Polimeni, J. R., Van Essen, D. C., \& Jenkinson, M. (2013). The minimal preprocessing pipelines for the Human Connectome Project. NeuroImage, 80, 105-124. https://doi.org/10.1016/j.neuroimage.2013.04.127 
Goulas, A., Margulies, D. S., Bezgin, G., \& Hilgetag, C. C. (2019). The architecture of mammalian cortical connectomes in light of the theory of the dual origin of the cerebral cortex. Cortex, 118, 244-261. https://doi.org/10.1016/j.cortex.2019.03.002

Greve, D. N., \& Fischl, B. (2009). Accurate and Robust Brain Image Alignment using Boundarybased $\quad$ Registration. $\quad$ NeuroImage, $\quad 48(1), \quad 63-72$. https://doi.org/10.1016/j.neuroimage.2009.06.060

Guell, X., Schmahmann, J. D., Gabrieli, J. D. D. E., \& Ghosh, S. S. (2018). Functional gradients of the cerebellum. ELife, 7. https://doi.org/10.7554/eLife.36652

Haak, K. V., \& Beckmann, C. F. (2020). Understanding brain organisation in the face of functional heterogeneity and functional multiplicity. NeuroImage, 220, 117061. https://doi.org/10.1016/j.neuroimage.2020.117061

Haak, K. V., Marquand, A. F., \& Beckmann, C. F. (2018). Connectopic mapping with resting-state fMRI. NeuroImage, 170, 83-94. https://doi.org/10.1016/J.NEUROIMAGE.2017.06.075

Haast, R. A. M., Ivanov, D., Formisano, E., \& Uludağ, K. (2016). Reproducibility and reliability of quantitative and weighted $\mathrm{T} 1$ and $\mathrm{T} 2 *$ mapping for myelin-based cortical parcellation at 7 Tesla. Frontiers in Neuroanatomy, 10, 112.

Hill, J., Inder, T., Neil, J., Dierker, D., Harwell, J., \& Essen, D. V. (2010). Similar patterns of cortical expansion during human development and evolution. Proceedings of the National Academy of Sciences, 107(29), 13135-13140. https://doi.org/10.1073/pnas.1001229107

Honey, C. J., Sporns, O., Cammoun, L., Gigandet, X., Thiran, J. P., Meuli, R., \& Hagmann, P. (2009). Predicting human resting-state functional connectivity from structural connectivity. Proceedings of the National Academy of Sciences, 106(6), 2035-2040. https://doi.org/10.1073/pnas.0811168106 
Hong, S.-J., Bernhardt, B. C., Gill, R. S., Bernasconi, N., \& Bernasconi, A. (2017). The spectrum of structural and functional network alterations in malformations of cortical development. Brain, 140(8), 2133-2143. https://doi.org/10.1093/brain/awx145

Hong, S.-J., Hyung, B., Paquola, C., \& Bernhardt, B. C. (2019). The Superficial White Matter in Autism and Its Role in Connectivity Anomalies and Symptom Severity. Cerebral Cortex, 29(10), 4415-4425. https://doi.org/10.1093/cercor/bhy321

Hong, S.-J., Vos de Wael, R., Bethlehem, R. A. I., Lariviere, S., Paquola, C., Valk, S. L., Milham, M. P., Di Martino, A., Margulies, D. S., Smallwood, J., \& Bernhardt, B. C. (2019). Atypical functional connectome hierarchy in autism. Nature Communications, 10(1), 1022. https://doi.org/10.1038/s41467-019-08944-1

Howells, H., Thiebaut de Schotten, M., Dell'Acqua, F., Beyh, A., Zappalà, G., Leslie, A., Simmons, A., Murphy, D. G., \& Catani, M. (2018). Frontoparietal Tracts Linked to Lateralized Hand Preference and Manual Specialization. Cerebral Cortex, 28(7), 24822494. https://doi.org/10.1093/cercor/bhy040

Huntenburg, J. M., Bazin, P. L., Goulas, A., Tardif, C. L., Villringer, A., \& Margulies, D. S. (2017). A Systematic Relationship Between Functional Connectivity and Intracortical Myelin in the Human Cerebral Cortex. Cerebral Cortex (New York, N.Y. : 1991), 27(2), 981-997. https://doi.org/10.1093/cercor/bhx030

Huntenburg, J. M., Bazin, P.-L., \& Margulies, D. S. (2018). Large-scale gradients in human cortical organization. Trends in Cognitive Sciences, 22(1), 21-31.

Ioannidis, J. P. (2005). Why most published research findings are false. PLoS Medicine, 2(8), e124. 
Jeurissen, B., Tournier, J. D., Dhollander, T., Connelly, A., \& Sijbers, J. (2014). Multi-tissue constrained spherical deconvolution for improved analysis of multi-shell diffusion MRI data. NeuroImage, 103, 411-426. https://doi.org/10.1016/j.neuroimage.2014.07.061

Krubitzer, L. (2007). The magnificent compromise: Cortical field evolution in mammals. Neuron, 56(2), 201-208.

Langs, G., Golland, P., \& Ghosh, S. S. (2015). Predicting activation across individuals with resting-state functional connectivity based multi-atlas label fusion. Lecture Notes in Computer Science (Including Subseries Lecture Notes in Artificial Intelligence and Lecture Notes in Bioinformatics), 9350, 313-320. https://doi.org/10.1007/978-3-319-24571-3_38

Larivière, S., Vos de Wael, R., Hong, S.-J., Paquola, C., Tavakol, S., Lowe, A. J., Schrader, D. V, \& Bernhardt, B. C. (2019). Multiscale Structure-Function Gradients in the Neonatal Connectome. Cerebral Cortex. https://doi.org/10.1093/cercor/bhz069

Larivière, S., Weng, Y., Vos de Wael, R., Royer, J., Frauscher, B., Wang, Z., Bernasconi, A., Bernasconi, N., Schrader, D. V., Zhang, Z., \& Bernhardt, B. C. (2020). Functional connectome contractions in temporal lobe epilepsy: Microstructural underpinnings and predictors of surgical outcome. Epilepsia, 61(6), 1221-1233. https://doi.org/10.1111/epi.16540

Li, Q., Tavakol, S., Royer, J., Larivière, S., Vos De Wael, R., Park, B., Paquola, C., Zeng, D., Caldairou, B., Bassett, D. S., Bernasconi, A., Bernasconi, N., Frauscher, B., Smallwood, J., Caciagli, L., Li, S., \& Bernhardt, B. C. (2020). Human brain function during pattern separation follows hippocampal and neocortical connectivity gradients. BioRxiv. https://doi.org/10.1101/2020.06.22.165290 
Liu, M., Bernhardt, B. C., Hong, S.-J., Caldairou, B., Bernasconi, A., \& Bernasconi, N. (2016). The superficial white matter in temporal lobe epilepsy: A key link between structural and functional network disruptions. Brain, 139(9), 2431-2440. https://doi.org/10.1093/brain/aww167

Lowe, A. J., Paquola, C., Vos de Wael, R., Girn, M., Lariviere, S., Tavakol, S., Caldairou, B., Royer, J., Schrader, D. V., Bernasconi, A., Bernasconi, N., Spreng, R. N., \& Bernhardt, B. C. (2019). Targeting age-related differences in brain and cognition with multimodal imaging and connectome topography profiling. Human Brain Mapping, 40(18), 52135230. https://doi.org/10.1002/hbm.24767

Margulies, D. S., Ghosh, S. S., Goulas, A., Falkiewicz, M., Huntenburg, J. M., Langs, G., Bezgin, G., Eickhoff, S. B., Castellanos, F. X., Petrides, M., Jefferies, E., \& Smallwood, J. (2016). Situating the default-mode network along a principal gradient of macroscale cortical organization. Proc. Natl. Acad. Sci. U. S. A., 113(44), 12574-12579. https://doi.org/10.1073/pnas.1608282113

Marques, J. P., Kober, T., Krueger, G., van der Zwaag, W., Van de Moortele, P.-F., \& Gruetter, R. (2010). MP2RAGE, a self bias-field corrected sequence for improved segmentation and T1-mapping at high field. Neuroimage, 49(2), 1271-1281.

Mars, R. B., Eichert, N., Jbabdi, S., Verhagen, L., \& Rushworth, M. F. S. (2018). Connectivity and the search for specializations in the language-capable brain. Current Opinion in Behavioral Sciences, 21, 19-26. https://doi.org/10.1016/j.cobeha.2017.11.001

Mars, R. B., Passingham, R. E., Neubert, F.-X., Verhagen, L., \& Sallet, J. (2017). 4.12Evolutionary Specializations of Human Association Cortex. In J. H. Kaas (Ed.), Evolution 
of Nervous Systems (Second Edition) (pp. 185-205). Academic Press. https://doi.org/10.1016/B978-0-12-804042-3.00118-4

Mars, R. B., Sallet, J., Neubert, F.-X., \& Rushworth, M. F. S. (2013). Connectivity profiles reveal the relationship between brain areas for social cognition in human and monkey temporoparietal cortex. Proceedings of the National Academy of Sciences, 110(26), 10806-10811. https://doi.org/10.1073/pnas.1302956110

Mars, R. B., Sotiropoulos, S. N., Passingham, R. E., Sallet, J., Verhagen, L., Khrapitchev, A. A., Sibson, N., \& Jbabdi, S. (2018). Whole brain comparative anatomy using connectivity blueprints. ELife, 7, e35237. https://doi.org/10.7554/eLife.35237

Mohedano-Moriano, A., Muñoz-López, M., Sanz-Arigita, E., Pró-Sistiaga, P., Martínez-Marcos, A., Legidos-Garcia, M. E., Insausti, A. M., Cebada-Sánchez, S., Arroyo-Jiménez, M. D. M., Marcos, P., Artacho-Pérula, E., \& Insausti, R. (2015). Prefrontal cortex afferents to the anterior temporal lobe in the Macaca fascicularis monkey. Journal of Comparative Neurology, 523(17), 2570-2598. https://doi.org/10.1002/cne.23805

Moonesinghe, R., Khoury, M. J., \& Janssens, A. C. J. W. (2007). Most Published Research Findings Are False-But a Little Replication Goes a Long Way. PLOS Medicine, 4(2), e28. https://doi.org/10.1371/journal.pmed.0040028

Morán, M. A., Mufson, E. J., \& Mesulam, M. -M. (1987). Neural inputs into the temporopolar cortex of the rhesus monkey. Journal of Comparative Neurology, 256(1), 88-103. https://doi.org/10.1002/cne.902560108

Mueller, S., Wang, D., Fox, M. D., Thomas Yeo, B. T., Sepulcre, J., Sabuncu, M. R., Shafee, R., Lu, J., \& Liu, H. (2013). Individual Variability in Functional Connectivity Architecture of the Human Brain. Neuron, 77(3), 586-595. https://doi.org/10.1016/j.neuron.2012.12.028 
Murphy, C., Wang, H.-T., Konu, D., Lowndes, R., Margulies, D. S., Jefferies, E., \& Smallwood, J. (2019). Modes of operation: A topographic neural gradient supporting stimulus dependent and independent cognition. NeuroImage, 186, 487-496.

Murphy, K., \& Fox, M. D. (2017). Towards a consensus regarding global signal regression for resting state functional connectivity MRI. NeuroImage, 154, 169-173. https://doi.org/10.1016/j.neuroimage.2016.11.052

Ocklenburg, S., Friedrich, P., Güntürkün, O., \& Genç, E. (2016). Intrahemispheric white matter asymmetries: The missing link between brain structure and functional lateralization? Reviews in the Neurosciences, 27(5), 465-480.

Oishi, K., Zilles, K., Amunts, K., Faria, A., Jiang, H., Li, X., Akhter, K., Hua, K., Woods, R., Toga, A. W., Pike, G. B., Rosa-Neto, P., Evans, A., Zhang, J., Huang, H., Miller, M. I., van Zijl, P. C. M., Mazziotta, J., \& Mori, S. (2008). Human brain white matter atlas: Identification and assignment of common anatomical structures in superficial white matter. NeuroImage, 43(3), 447-457. https://doi.org/10.1016/j.neuroimage.2008.07.009

Open Science Collaboration. (2015). Estimating the reproducibility of psychological science. Science, 349(6251).

Paquola, C., Vos De Wael, R., Wagstyl, K., Bethlehem, R. A. I., Hong, S.-J., Seidlitz, J., Bullmore, E. T., Evans, A. C., Misic, B., Margulies, D. S., Smallwood, J., \& Bernhardt, B. C. (2019). Microstructural and functional gradients are increasingly dissociated in transmodal cortices. PLOS Biology, 17(5), e3000284. https://doi.org/10.1371/journal.pbio.3000284

Park, B., Bethlehem, R. A. I., Paquola, C., Larivière, S., Cruces, R. R., Vos de Wael, R., Consortium, N. in P. N. (NSPN), Bullmore, E. T., \& Bernhardt, B. C. (2020). Macroscale 
connectome manifold expansion in adolescence. BioRxiv, 2020.06.22.165621. https://doi.org/10.1101/2020.06.22.165621

Park, B., Hong, S.-J., Valk, S. L., Paquola, C., Benkarim, O., Bethlehem, R. A., Di Martino, A., Milham, M., Gozzi, A., Yeo, B. T., \& others. (2020). Connectome and microcircuit models implicate atypical subcortico-cortical interactions in autism pathophysiology. BioRxiv.

Park, B., Vos de Wael, R., Paquola, C., Larivière, S., Benkarim, O., Royer, J., Tavakol, S., Cruces, R. R., Li, Q., Valk, S. L., Margulies, D. S., Mišić, B., Bzdok, D., Smallwood, J., \& Bernhardt, B. C. (2021). Signal diffusion along connectome gradients and inter-hub routing differentially contribute to dynamic human brain function. NeuroImage, 224, 117429. https://doi.org/10.1016/j.neuroimage.2020.117429

Patel, G. H., Yang, D., Jamerson, E. C., Snyder, L. H., Corbetta, M., \& Ferrera, V. P. (2015). Functional evolution of new and expanded attention networks in humans. Proceedings of the National Academy of Sciences, 112(30), 9454-9459. https://doi.org/10.1073/pnas.1420395112

Phelps, E. A. (2004). Human emotion and memory: Interactions of the amygdala and hippocampal complex. Current Opinion in Neurobiology, 14(2), 198-202. https://doi.org/10.1016/j.conb.2004.03.015

Ralph, M. A. L., Jefferies, E., Patterson, K., \& Rogers, T. T. (2017). The neural and computational bases of semantic cognition. Nature Reviews Neuroscience, 18(1), 42.

Rilling, J. K., Glasser, M. F., Preuss, T. M., Ma, X., Zhao, T., Hu, X., \& Behrens, T. E. J. (2008). The evolution of the arcuate fasciculus revealed with comparative DTI. Nature Neuroscience, 11(4), 426-428. https://doi.org/10.1038/nn2072 
Robinson, E. C., Jbabdi, S., Glasser, M. F., Andersson, J., Burgess, G. C., Harms, M. P., Smith, S. M., Van Essen, D. C., \& Jenkinson, M. (2014). MSM: A new flexible framework for Multimodal Surface Matching. NeuroImage, $\quad 100, \quad 414-426$. https://doi.org/10.1016/j.neuroimage.2014.05.069

Roumazeilles, L., Eichert, N., Bryant, K. L., Folloni, D., Sallet, J., Vijayakumar, S., Foxley, S., Tendler, B. C., Jbabdi, S., Reveley, C., Verhagen, L., Dershowitz, L. B., Guthrie, M., Flach, E., Miller, K. L., \& Mars, R. B. (2020). Longitudinal connections and the organization of the temporal cortex in macaques, great apes, and humans. PLOS Biology, 18(7), e3000810. https://doi.org/10.1371/journal.pbio.3000810

Sakata, H., Kim, Y., Nejime, M., Konoike, N., Miyachi, S., \& Nakamura, K. (2019). Laminar Pattern of Projections Indicates the Hierarchical Organization of the Anterior CingulateTemporal Lobe Emotion System. Frontiers in Neuroanatomy, 13. https://doi.org/10.3389/fnana.2019.00074

Salimi-Khorshidi, G., Douaud, G., Beckmann, C. F., Glasser, M. F., Griffanti, L., \& Smith, S. M. (2014). Automatic denoising of functional MRI data: Combining independent component analysis and hierarchical fusion of classifiers. NeuroImage, 90, 449-468. https://doi.org/10.1016/j.neuroimage.2013.11.046

Sanides, F. (1969). Comparative architectonics of the neocortex of mammals and their evolutionary interpretation. Annals of the New York Academy of Sciences, 167(1), 404423.

Saur, D., Kreher, B. W., Schnell, S., Kümmerer, D., Kellmeyer, P., Vry, M.-S., Umarova, R., Musso, M., Glauche, V., Abel, S., Huber, W., Rijntjes, M., Hennig, J., \& Weiller, C. 
(2008). Ventral and dorsal pathways for language. Proceedings of the National Academy of Sciences, 105(46), 18035. https://doi.org/10.1073/pnas.0805234105

Scannell, J. W., Blakemore, C., \& Young, M. P. (1995). Analysis of connectivity in the cat cerebral cortex. The Journal of Neuroscience: The Official Journal of the Society for Neuroscience, $15(2), 1463-1483$.

Schilling, K., Gao, Y., Janve, V., Stepniewska, I., Landman, B. A., \& Anderson, A. W. (2018). Confirmation of a gyral bias in diffusion MRI fiber tractography. Human Brain Mapping, 39(3), 1449-1466. https://doi.org/10.1002/hbm.23936

Smiley, J. F., \& Falchier, A. (2009). Multisensory connections of monkey auditory cerebral cortex. Hearing Research, 258(1-2), 37-46. https://doi.org/10.1016/j.heares.2009.06.019

Smith, R. E., Tournier, J. D., \& Calamante, F. (2012). Anatomically-constrained tractography: Improved diffusion MRI streamlines tractography through effective use of anatomical information. $\quad$ NeuroImage, $\quad$ 62(3), $\quad$ 1924-1938. https://doi.org/10.1016/j.neuroimage.2012.06.005

Smith, R. E., Tournier, J. D., Calamante, F., \& Connelly, A. (2015a). SIFT2: Enabling dense quantitative assessment of brain white matter connectivity using streamlines tractography. NeuroImage, 119, 338-351. https://doi.org/10.1016/j.neuroimage.2015.06.092

Smith, R. E., Tournier, J.-D., Calamante, F., \& Connelly, A. (2015b). The effects of SIFT on the reproducibility and biological accuracy of the structural connectome. NeuroImage, 104, 253-265. https://doi.org/10.1016/j.neuroimage.2014.10.004

Smith, S. M., Jenkinson, M., Woolrich, M. W., Beckmann, C. F., Behrens, T. E., Johansen-Berg, H., Bannister, P. R., De Luca, M., Drobnjak, I., Flitney, D. E., \& others. (2004). Advances 
in functional and structural MR image analysis and implementation as FSL. NeuroImage, 23, S208-S219.

Suárez, L. E., Markello, R. D., Betzel, R. F., \& Misic, B. (2020). Linking Structure and Function in Macroscale Brain Networks. Trends in Cognitive Sciences, 24(4), 302-315. https://doi.org/10.1016/j.tics.2020.01.008

Tournier, J. D., Calamante, F., \& Connelly, A. (2010). Improved probabilistic streamlines tractography by 2 nd order integration over fibre orientation distributions. Proceedings of the 18th Annual Meeting of the International Society for Magnetic Resonance in Medicine (ISMRM). https://doi.org/10.1103/PhysRevLett.116.058301

Tournier, J. D., Calamante, F., \& Connelly, A. (2012). MRtrix: Diffusion tractography in crossing fiber regions. International Journal of Imaging Systems and Technology, 22(1), 53-66. https://doi.org/10.1002/ima.22005

Valk, S. L., Xu, T., Margulies, D. S., Masouleh, S. K., Paquola, C., Goulas, A., Kochunov, P., Smallwood, J., Yeo, B. T. T., Bernhardt, B. C., \& Eickhoff, S. B. (2020). Shaping Brain Structure: Genetic and Phylogenetic Axes of Macro Scale Organization of Cortical Thickness [Preprint]. Neuroscience. https://doi.org/10.1101/2020.02.10.939561

Van Essen, D. C., Smith, S. M., Barch, D. M., Behrens, T. E. J., Yacoub, E., Ugurbil, K., \& Consortium, W. U.-M. H. C. P. (2013). The WU-Minn Human Connectome Project: An overview. NeuroImage, 80, 62-79. https://doi.org/10.1016/j.neuroimage.2013.05.041

Vaz, A. P., Inati, S. K., Brunel, N., \& Zaghloul, K. A. (2019). Coupled ripple oscillations between the medial temporal lobe and neocortex retrieve human memory. Science, 363(6430), 975978. https://doi.org/10.1126/science.aau8956 
Vos de Wael, R., Benkarim, O., Paquola, C., Lariviere, S., Royer, J., Tavakol, S., Xu, T., Hong, S.-J., Langs, G., Valk, S., \& others. (2020). BrainSpace: A toolbox for the analysis of macroscale gradients in neuroimaging and connectomics datasets. Communications Biology, 3(1), 1-10.

Vos de Wael, R., Hyder, F., \& Thompson, G. J. (2017). Effects of Tissue-Specific Functional Magnetic Resonance Imaging Signal Regression on Resting-State Functional Connectivity. Brain Connectivity, 7(8), 482-490. https://doi.org/10.1089/brain.2016.0465

Vos de Wael, R., Larivière, S., Caldairou, B., Hong, S.-J., Margulies, D. S., Jefferies, E., Bernasconi, A., Smallwood, J., Bernasconi, N., \& Bernhardt, B. C. (2018). Anatomical and microstructural determinants of hippocampal subfield functional connectome embedding. Proceedings of the National Academy of Sciences, 115(40), 201803667. https://doi.org/10.1073/pnas.1803667115

Wagner, H. H., \& Dray, S. (2015). Generating spatially constrained null models for irregularly spaced data using Moran spectral randomization methods. Methods in Ecology and Evolution, 6(10), 1169-1178. https://doi.org/10.1111/2041-210X.12407

Wang, P., Kong, R., Kong, X., Liégeois, R., Orban, C., Deco, G., Van Den Heuvel, M. P., \& Yeo, B. T. T. (2019). Inversion of a large-scale circuit model reveals a cortical hierarchy in the dynamic resting human brain. Science Advances, 5(1). https://doi.org/10.1126/sciadv.aat7854

Webster, M., Ungerleider, L., \& Bachevalier, J. (1991). Connections of inferior temporal areas TE and TEO with medial temporal-lobe structures in infant and adult monkeys. Journal of Neuroscience, 11(4), 1095-1116. 
Worsley, K. J., Taylor, J. E., Carbonell, F., Chung, M., Duerden, E., Bernhardt, B., Lyttelton, O., Boucher, M., \& Evans, A. (2009). SurfStat: A Matlab toolbox for the statistical analysis of univariate and multivariate surface and volumetric data using linear mixed effects models and random field theory. Neuroimage, 47, S102.

Xu, T., Nenning, K.-H., Schwartz, E., Hong, S.-J., Vogelstein, J. T., Goulas, A., Fair, D. A., Schroeder, C. E., Margulies, D. S., Smallwood, J., Milham, M. P., \& Langs, G. (2020). Cross-species functional alignment reveals evolutionary hierarchy within the connectome. NeuroImage, 223, 117346. https://doi.org/10.1016/j.neuroimage.2020.117346

Yarkoni, T., Poldrack, R. A., Nichols, T. E., Van Essen, D. C., \& Wager, T. D. (2011). Large-scale automated synthesis of human functional neuroimaging data. Nature Methods, 8(8), 665670. https://doi.org/10.1038/nmeth.1635

Yeh, C.-H., Smith, R. E., Liang, X., Calamante, F., \& Connelly, A. (2016). Correction for diffusion MRI fibre tracking biases: The consequences for structural connectomic metrics. NeuroImage, 142, 150-162. https://doi.org/10.1016/j.neuroimage.2016.05.047

Yeo, B. T. T., Krienen, F. M., Sepulcre, J., Sabuncu, M. R., Lashkari, D., Hollinshead, M., Roffman, J. L., Smoller, J. W., Zöllei, L., Polimeni, J. R., Fischl, B., Liu, H., \& Buckner, R. L. (2011). The organization of the human cerebral cortex estimated by intrinsic functional connectivity. J. Neurophysiol., 106(3), 1125-1165. https://doi.org/10.1152/jn.00338.2011

Young, M. P. (1992). Objective analysis of the topological organization of the primate cortical visual system. Nature, 358(6382), 152-155. 